\title{
Mental Disordersand Suicide
}

\author{
Susheelkumar V Ronad ${ }^{1 *}$, Kirankumar TC ${ }^{2}$, Pankaja TC ${ }^{3}$ and Santosh S Ugargol ${ }^{4}$ \\ ${ }^{1}$ Department of Psychiatric Nursing, Dimhans Dharwad, India
}

${ }^{2}$ Department of Management Studies, Karnataka Arts College Dharwad, India

${ }^{3}$ Assistant Professor, Rl Law College Davangere, India

${ }^{4}$ First Year Msc Nursing (Psychiatry), Dimhans Dharwad, India

Received: September 14, 2017; Published: September 27, 2017

*Corresponding author: Susheel kumar V Ronad, Assistant professor, Department of psychiatric nursing, Dimhans Dharwad, Karnataka, India, Email: susheelronad@gmail.com

\section{Introduction}

This section takes a look at some of the major types of mental disorders and describes how they may increase the risk of suicidal behavior in persons with these disorders. Included in this discussion are the principal disorders-depression, bipolar disorder, and schizophrenia; in addition, the relationships between suicide and postpartum depression, eating disorders, self-mutilation, posttraumatic stress disorder, and alcohol/substance abuse are briefly considered.

With each of these disorders there is great need to provide information to the general public about the symptoms of the disease, its epidemiology, risk factors, clinical best practice, and research needs. Mental health education and awareness efforts also need to provide information on the various options for mental health treatment and their efficacy in reducing risk of suicide, the debate surrounding the safety of selective serotonin reuptake inhibitors (SSRIs), and the need to balance the possible benefits and risks in the selection of any method of treatment for mental illnesses.

\section{Suicide and Depression}

Depression is a very common mental health problem worldwide. The World Health Organization estimates that 121 million people currently suffer from depression, with 5.8\% of men and 9.5\% of women experiencing a depressive episode in any given year [1]. It is estimated that depression will become the second most common cause of disability, after heart disease, by 2020 [2] Women are twice as likely to be diagnosed and treated for depression. However, it is believed that men suffer depression to a larger extent than the statistics show, since men are less likely to seek medical help and when they do, doctors are less likely to detect depressive symptoms [3] In light of these high rates of depression, it is a cause for concern that mood disorders (of which depression is the major example) are the most common psychiatric condition associated with suicide [4]. It is important to note, however, that depression encompasses a wide range of experiences and illness forms from mild to severe, transient to permanent, and the risk of suicide varies substantially with the type of depression.

Amongst those diagnosed with depression, a study in Finland [5] found that key indicators for suicide include: previous selfharm, severity of the illness, alcohol or drug abuse, serious or chronic physical illnesses, lack of a partner, anxiety and personality disorders. Given the high prevalence of suicide in the community, and the high rates of depression amongst those who die by suicide, it is vital that those who are suffering from depression seek effective treatment that addresses both depression and suicidality. Since there is no evidence that antidepressant medications alone reduce suicide risk, practitioners' ability to recognize and address suicidality can be a life-saving skill. Since males in the industrialized nations are at higher risk for suicide and are less likely than females to seek medical attention for depression, considering alternative settings to reach at-risk males may require innovative approaches by communities (e.g., providing hot-line numbers in pubs).

\section{Distinguishing Between Depression and Normal States of Sadness and Grief}

Experts report that from $20 \%$ to $60 \%$ of the deaths by suicide occur among people who have a mood disorder. Suicide among such people is more common among those with more severe and/or psychotic symptoms, with late onset, and with coexisting metal and addictive disorders. Death through suicide is also more common among those who have experienced stressful life events, who have a medical illness and who have a family history of suicide. The sadness of a major depressive disorder differs both in degree and amount from the sadness that strikes anyone at times when life is especially hard. Normal states of grief or sadness generally have less pervasive effects and last for shorter periods of time than those that mark major depression. Furthermore, certain symptoms of severe depression only rarely occur in those experiencing times of normal sadness. 
These include anhedonia (the inability to experience pleasure), hopelessness, and loss of mood reactivity (the ability to feel a mood uplift in response to something positive). Suicidal thoughts and psychotic symptoms, when associated with depressive symptoms, usually mean that a person truly does have a diagnosable depressive disorder. Nevertheless, many symptoms commonly tied to major depressive disorders do also occur during times of severe stress. Among them: sleep disturbances, changes in appetite, and troubles with concentration, brooding on sad thoughts or feelings, or substance use. When a person with these kinds of problems sees a healthcare provider, the challenge to that professional is to distinguish normal sadness from pathological states and then to provide treatment.

\section{Suicide and Postpartum Depression}

It is somewhat startling that in many countries suicide is now the leading external cause of death amongst new mothers [6]. In most of these cases, women are suffering with postpartum depression. Between $8-15 \%$ of women suffer postpartum depression, and the condition is usually mild and manageable. Severe postpartum depression, however, is linked to an elevated suicide risk, with those who are admitted to hospital up to 70 times as likely to die by suicide [7]. Risks are especially high in the first year after childbirth. The huge stigma that women suffering from postpartum depression face must be tackled if suicide rates in this high-risk group are to be reduced. Recent developments including a number of high profile celebrities (including Brooke Shields, Natasha Hamilton and Elle Macpherson) talking about their experiences with postpartum depression is to be applauded, but more needs to be done. In particular it is important that high-risk women are identified during pregnancy and adequate preparations are made for their care.

Furthermore, women presenting with postpartum depression must not slip through the cracks in primary health care clinics. One recent study found that only $29 \%$ of women presenting with high levels of depressive symptoms on the psychiatric symptom index were diagnosed with postpartum depression [8]. Increasing this figure might require changes in the way medical professionals are trained to identify this condition. Once diagnosed, there are a host of effective pharmacological and psychotherapeutic treatment options whose efficacy has been well documented $[9,10]$.

\section{Suicide and Bipolar Disorder}

Bipolar affective disorder is a common condition, and among mental illnesses ranks second only to unipolar depression as a cause of worldwide disability [11]. Bipolar depression affects men and women equally, and afflicts about 5 people in 1,000 [12] for those with bipolar depression, suicide risks are approximately 15 times that of the general population [13]. Suicide often first occurs when work, study, family or emotional pressures are at their greatest. In women, suicidal risks can increase postpartum or during menopause. Most persons with bipolar affective disorder have the potential, with optimal treatment, to return to normal functioning. With sub-optimal treatment, however, many will have a poor long term outcome with increased risk of suicide. Yet there is evidence that treatment is generally sub optimal.

Longitudinal observational studies suggest that the very high lifetime prevalence of suicide attempts in people with bipolar affective disorder (50\%) [14] Could be reduced by maintenance drug treatment and adequate treatment of depression and co morbid alcohol and drug abuse [15]. Adequate treatment goes beyond the simple provision of treatment regimes, and it is at these times that suicide risk is particularly high. People with bipolar disorders need encouragement to initiate and stick to treatment regimes and continued follow-up of their treatment. Despite its shortcomings, lithium has long been the mainstay of treatment for bipolar affective disorder. Several newer drugs have emerged over the past 10 years, but evidence of their suicidal protection effectiveness remains sparse.

\section{Suicide and Schizophrenia}

Approximately 24 million people worldwide suffer from schizophrenia, with equal rates (7 per 1000) reported amongst men and women [16]. It is estimated that there is a 4 to 10 percent lifetime risk for suicide among persons with schizophrenia and a $40 \%$ lifetime risk of suicide attempts [17]. A World Health Organization study found the most common cause of death in those with schizophrenia was suicide [18].

Risk factors for suicide amongst those suffering from schizophrenia include: positive symptoms, co-morbidity with depression, lack of treatment, downgrading in level of care, chronic illness, a good educational background and high performance expectations. Suicide is more likely to occur earlier in the course of the illness. If we are to prevent suicide in persons with schizophrenia it is vital that all staff working in mental health receive dedicated training in both risk assessment and management, something that is too often left to experience to provide [19]. Training should also emphasize the importance of addressing co-morbid conditions that have been found to heighten risk, such as depression, substance use and loss of functioning. The simple measure of improving record keeping and care plans may help ensure the entire clinical team is aware of all the risk factors present and how they can be minimized. This also highlights the need for good communication between the patient's multidisciplinary team, general practitioner, caregivers and relatives.

\section{Preventing Suicide in People Who Have Schizophrenia}

The future holds better treatments for people with schizophrenia. It is important for both caregivers and loved ones of people with this disease to encourage them to hold on to life. Emphasize that improved treatments promise to emerge from scientific laboratories in years to come. There is real reason to expect that life will get better. In 2005, the British Journal of Psychiatry published an analysis that combined a large number of international studies of suicide risk among this population. The most robust finding of this review was that suicide was more likely when people with schizophrenia showed agitation and expressed 
feelings of worthlessness and/or hopelessness; also when they had a history of suicidal thoughts and of suicide attempts. A family history of suicide also raised the risk.

\section{Among the other important findings of this study:}

The living situation of a person with schizophrenia affects risk. Those who live alone, or do not live with their families, are at increased risk of suicide. Recent losses (such as a divorce or death) increases risk, as is true for the general population. Those individuals who are better educated seem to be at higher risk for suicide. This may reflect the greater awareness of and fear of mental deterioration. Poor adherence to treatment greatly increases risk. In some patients the use of medications known to have antisuicide effects may be advisable. Alcohol misuse did not appear to be a major risk factor for people with schizophrenia. However, abuse of drugs was strongly correlated with heightened risk. Such drug abuse is twice as common among those with schizophrenia compared to the general population.

\section{High-Risk Times for Suicide}

People with schizophrenia need extra support and supervision at these times: Periods when the person is very psychotic and out of touch with reality Periods when they are very depressed In the first 6 to 9 months after they have started first taking medications, when they are thinking more clearly and learn that they have schizophrenia (with all the negative implications of this) The period after hospital discharge. Discharge plans ought to be made with care. Whenever possible, a person at high risk ought not to be left alone, and certainly not for long periods. Suicide often occurs when a person with schizophrenia has been left alone all day.

Anorexia Nervosa and Bulimia Nervosa: Eating disorders have the highest mortality rate of any mental illness. This includes both suicide deaths and deaths from direct complications of their eating disorder. Studies have found that prevalence rates for attempted suicide vary depending on the eating disorder diagnostic subgroup and study setting. The prevalence of suicide attempts is lowest among outpatients with anorexia nervosa (16\%). Prevalence rates are higher for bulimic individuals treated as outpatients (23\%) and inpatients (39\%). The highest rates of suicide attempts are reported among bulimic individuals who have co-morbid alcohol abuse (54\%) [20]. It should be noted that these rates do not include those who die from other complications of the disorder.

Further complicating this picture is the high prevalence of non-lethal self-mutilation amongst those diagnosed with eating disorders. Unsurprisingly, most of the women in the study had other psychiatric disorders besides an eating disorder, including depression, drug or alcohol abuse or fearfulness or anxiety. Almost 84 percent of the patients had at least one other psychiatric problem. Given these statistics it is concerning that the incidence of eating disorders has doubled since the 1960s. Eating disorders, which used to be a largely Western disorder, are also being reported in high levels in many Asian countries for the first time [21,22]. More than $90 \%$ of those diagnosed each year are young women Dr.
Mike Shooter, president of the Royal College of Psychiatrists has argued that eating disorders are "poorly understood by the public and clinicians alike and that there is a need for intensive research into clinical best practice guidelines.

\section{Self-Mutilation}

Self mutilation, especially among young people, is an emerging area of concern. One widely cited estimate is that self-mutilation occurs in at least 1 person per 1,000 annually. Self-mutilation takes many forms and can include self binding and amputation, banging ones head, biting oneself, pulling ones hair, and cutting, scratching or burning ones skin. Self-mutilation should not be confused with suicide attempts, but research suggests that those with mental illnesses are more likely to self-mutilate, with one study of psychiatric outpatients finding that 33\% reported engaging in selfharm in the previous three months. Furthermore, self-mutilation is an early predictor of suicidal behavior. About half of all people who kill themselves have a history of deliberate self-harm, an episode having occurred within the year before death in $20-25 \%$.

\section{Substance Abuse and Suicide}

International health researchers point to alcohol as the most widely abused substances in the world. Globally, the extent of problem drinking varies widely; overall about $1.7 \%$ of the world's people abuse alcohol. In parts of Eastern Europe and in North America, experts estimate that $5 \%$ of the population abuse alcohol. The prevalence of illegal abuse of drugs and drug addiction in the worlds' nations ranges from $0.4 \%$ to $4 \%$. In 2003, the World Health Organization estimated about 5 million people inject illicit drugs. In studies that examine risk factors among people lost to suicide, substance abuse and problem drinking occur more often among youths and younger adults, compared to older people. Also, male gender is a risk factor for both substance abuse and suicide. For particular groups at risk, such as indigenous peoples surrounded by an alien majority culture, depression and alcohol abuse may be co-existing risk factors for suicide.

Alcohol and substance abuse problems contribute to suicidal behavior in several ways. Persons who use and abuse substances often have several other risk factors for suicide. In addition to being depressed, they are also likely to have social and financial problems. Substance use and abuse tend to occur more often among people who are prone to act on impulse. They also occur often among those who engage in many types of high-risk behaviours that can cause self-harm. In addition, people who are intoxicated may make suicide attempts impulsively and aggressively that they would not make if they were not intoxicated.

\section{Key Facts about Substance Abuse and Suicide}

a. People with severe alcohol dependency or alcoholism have increased risk for suicide.

b. Depression and other mood disorders are involved with the majority of suicides; substance abusers have profoundly increased rates of depression. Left untreated, substance abuse worsens the outcomes of mood disorders. 
c. Suicide attempts occur more often within the context of a binge-drinking episode.

d. People whose drinking causes trouble at work are six times as likely as others to die by suicide in the home.

e. Problem drinkers who have been hospitalized for reasons related to alcohol abuse have ten times the risk of suicide, compared to problem drinkers not so hospitalized. Substance dependence raises the risk of work, family and physical health problems that can become more severe over time.

f. If a person who is dependent on alcohol also uses cocaine the risk rises significantly.

g. Places that have higher per capita spirits sales tend to have higher suicide rates.

h. Alcoholics who die by suicide are more likely to have partner-relationship troubles and other severe life stressors than alcoholics who have not tried suicide.

i. Canadian research indicates that up to $80 \%$ of people with schizophrenia will abuse substances at some time; such abuse is associated with suicidal behavior.

j. High-risk alcoholics can be defined as those with a cooccurring depression diagnosis, those in treatment for a prior suicide attempt or those who have tried to ill themselves in the past.

k. The risk of suicide among alcoholics increases over time; suicide risk is highest after 10 or more years of having drinking problems.

\section{Treating Mental Illnesses including Substance Abuse Disorders Lowers Risk}

There are effective ways to lower the risk of problem drinking and drug abuse among young people. There are also effective treatments, which often combine medications with various forms of psychotherapy and support groups, for alcoholism and substance abuse. New treatments, tailored to people with both substance abuse problems and suicidal tendencies, are forthcoming. These may offer greater promise for saving many individuals. People who drink too much often seem to die by suicide in the throes of severe relationships difficulties. Accordingly, for people at risk of suicide who drink too much, marital or couples based treatment is showing particular promise. Alcohol and substance abuse clinicians and counsellors need training in and an understanding of depression and other mental illnesses that can lead to suicide. If these caregivers can better recognize mental illnesses, it will help them better treat people with alcoholism.

\section{References}

1. (2001) World Health Organization 2001, "Mental and Neurological Disorders," Fact sheet No. 265.
2. Peveler R, Alan Carson, Gary Rodin (2002) "ABC of Psychological Medicine: Depression in Medical Patients," British Medical Journal 325(7356): 149-152.

3. Sane (2000) "Depression and Manic Depression".

4. Jamison KR (2000) "Suicide and Bipolar Disorder." Journal of Clinical Psychiatry 61(9): 47-51.

5. Petteri Sokero T, Tarja k Melartin, Heikki J Rytsälä, Ulla S Leskelä, Paula S Lestelä Mielonen, et al. (2005) "Prospective Study of Risk Factors for Attempted Suicide among Patients with DSM-IV Major Depressive Disorder." British Journal of Psychiatry 186: 314-318.

6. (2002) Royal College of Psychiatrists press release (2002) "Suicide Leading Cause of Death among New Mothers."

7. Appleby L, Mortensen PB, Faragher EB (1998) “Suicide and Other Causes of Mortality after Post-partum Psychiatric Admission," British Journal of Psychiatry 173: 209-211.

8. Heneghan AM, Silver EJ, Bauman LJ, Stein RE (2000) “Do Pediatricians Recognize Mothers with Depressive Symptoms?" Pediatrics 106: 13671373.

9. Appleby L, Warner R, Whitton A, Faragher B (1997) "A Controlled Study of Fluoxetine and Cognitive-Behavioural Counselling in the Treatment of Postnatal Depression." British Medical Journal 314(7085): 932-936.

10. Chabrol H, Teissedre F, Saint Jean M, Teisseyre N, Rogé B, et al. (2002) "Prevention and Treatment of Post-partum Depression: A Controlled Randomized Study on Women at Risk," Psychological Medicine 32(6): 1039-1047.

11. Murray CJ, Lopez AD (1997) "Global Mortality, Disability, and the Contribution of Risk Factors: Global Burden of Disease Study." Lancet 349(9063): 1436-1442.

12. Sane (2000) "Depression and Manic Depression."

13. Harris EC, B Barraclough (1997) "Suicide as an Outcome for Mental Disorders." British Journal of Psychiatry 170: 205-228.

14. (2001) Manic Depression Fellowship (2001) "Users Survey of Experiences of Health Services."

15. Tondo L, Baldessarini RJ, Hennen J, Floris G, Silvetti F, et al. (1998) "Lithium Treatment and Risk of Suicidal Behavior in Bipolar Disorder Patients." Journal of Clinical Psychiatry 59(8): 405-414.

16. (2001) World Health Organization (2001) "Mental and Neurological Disorders," Fact sheet No. 265.

17. World Fellowship for Schizophrenia and Allied Disorders, "Schizophrenia and Suicide," Pamphlet No 23.

18. Sartorius N, Jablensky A, Korten A, Ernberg G, Anker M, et al. (1986) "Early Manifestations and First Contact Incidence of Schizophrenia in Different Cultures." Psychological Medicine 16(4): 909-928.

19. Raymont V (2001) "Suicide in Schizophrenia-How Can Research Influence Training and Clinical Practice?" Psychiatric Bulletin 25(2): 4650.

20. Sansone RA, Levitt JL (2002) "Self-Harm Behaviors among Those with Eating Disorders: An Overview," Eating Disorders 10: 205-213.

21. Lee S (2000) “Eating Disorders Are Becoming More Common in the East Too," Letter to the Editor, British Medical Journal 321(7267): 1023.

22. Eating Disorders Coalition, "Statistics," www.eatingdisorderscoalition. org 


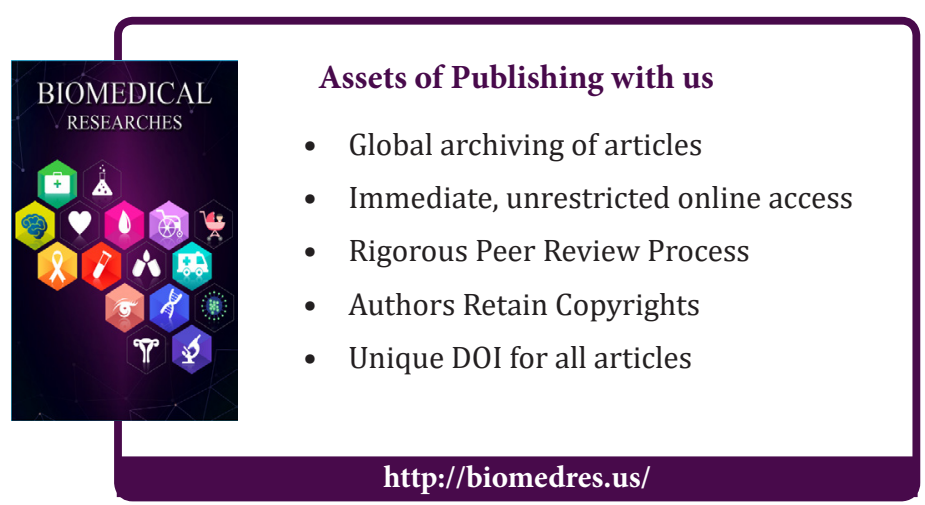

